\title{
The Causal Interactions Between Bilateral M1 and SMA During Verb Comprehension, Motor Imagery and Hand Motion
}

\author{
Jie Yang ${ }^{1, *} ;$ Hua Shu $^{2}$ \\ ${ }^{1}$ Department of Neurology, University of California Irvine, Irvine, USA \\ ${ }^{2}$ Key Laboratory of Cognitive Neuroscience and Learning, Beijing Normal University, Beijing, China \\ *Corresponding author: Jie Yang, Department of Neurology, University of California Irvine, Irvine, CA 92697, USA. Tel: +1-9178601890, Fax:+1-9498248549, E-mail: jiey7@uci.edu
}

Received: December 01, 2013; Revised: December 15, 2013; Accepted: December 16, 2013

\begin{abstract}
Background:The role of the sensory-motor system in semantic representation has caused increasing research interest. Although previous studies have shown different activations in the motor system during action verb processing, motor imagery and action execution, whether motor system interaction is modulated by task demand is still unknown.

Objectives: To address the issue, the current study used effective connectivity analysis to investigate how task demand influences causal interactions among bilateral supplementary motor areas (SMA) and primary motor cortex (M1).

Materials and Methods: Nineteen participants performed a verb reading task, a motor imagery task, and a hand motion task in an fMRI experiment, and their brain activity related to task performances was examined. Granger causality analysis was used to compute causal interactions among activity in the bilateral M1 and bilateral SMA in three tasks.

Results: Granger causality analysis indicated that the hand motion task elicited the most complicated network and that the passive reading task elicited the fewest connections among the four areas. Furthermore, only the passive reading task elicited left lateralized connectivity between M1 and SMA. In addition, motor imagery and hand motion execution elicited negative influences from the right SMA to other areas.

Conclusions:These results together suggest that although the motor network involved in action verb processing shared some interactions with those in motor imagery and hand motion execution, semantic representation during language comprehension is different from the raw sensory-motor experiences supported by the primary cortical areas.
\end{abstract}

Keywords:Brain; Comprehension; Imagery (Psychotherapy); Language; Magnetic Resonance Imaging; Motor Activity; Motor Cortex

\section{Background}

The role of sensory-motor system in semantic representation is highly controversial. So far various theories try to address the issue (1). Theories about strong embodiment claim that low-level sensory and motor information is activated in primary cortical areas as part of semantic processing (2-4). For example, Gallese and Lakoff proposed that the same neural substrates are used for perceiving/ doing, imaging, and language comprehension (5). This means that the primary motor cortex (M1) plays similar roles in action language comprehension, motor imagery, and action execution. Different from strong embodiment, weak embodiment claims that the sensory-motor system can partly contribute to semantic processing (6, 7). Moreover, semantic representation is different from raw sensory-motor experiences, and hence the roles of M1 in action language processing are different in motor imagery and action execution.

Several studies have indicated that M1 is activated dur- ing action language comprehension (8-13), and these findings support the strong embodiment view that raw sensory-motor information are used to represent semantic information. However, researchers also have found that the involvements of sensory-motor areas in action language comprehension and motor imagery are different. For instance, Willems et al. showed that in a motor imagery task, manual verbs elicited stronger activities in the primary motor cortex than did non-action verbs, whereas in a lexical decision task the manual verbs merely showed stronger effects in areas of premotor cortex. In addition, there was no overlap or correlation between the premotor effects elicited by the two tasks (14). The results suggest that the motor system plays different roles in action verb processing and motor imagery: in motor imagery the motor system is responsible for motor planning and execution, but in action verb comprehension the motor system might be only responsible for motor planning.

Implication for health policy/practice/research/medical education:

The current finding indicate that although the motor network involved in action verb processing shared similar interactions with those in motor imagery and hand motion execution, there were less connections within the motor system and the network was less complicated. This helps clarify the nature of embodied semantic representation.

Copyright @ 2014, Tehran University of Medical Sciences. This is an open-access article distributed under the terms of the Creative Commons Attribution License, which permits unrestricted use, distribution, and reproduction in any medium, provided the original work is properly cited. 
The above studies focus on the extent of brain activations in the motor system during action language comprehension and motor imagery, but whether interaction within the motor system is modulated by task demand (e.g. action verb processing, motor imagery, and hand motion execution) is still unclear. One promising approach to address the issue is effective connectivity analysis, which computes a directional influence of one neural system over another (15).

So far there are several methods to achieve effective connectivity analysis. One method is hypothesis-driven approaches, such as structural equation modeling (SEM). SEM is a multivariate regression analysis used to detect contemporaneous interactions among variables (16). In SEM, seed regions and their connections are specified by the user, and the path coefficient at each connection and model fit indices are obtained (17). Another method is data-driven analysis, such as Granger causality (GC) $(18,19)$. In GC, if the past information of a variable X can help predict the future of a variable $\mathrm{Y}$ with better accuracy, then the variable $\mathrm{X}$ is thought to "Granger cause" the variable Y (19). In fMRI, vector autoregressive (VAR) analysis is used to achieve GC modeling, and the delayed effects of one or multiple lags are used to calculate the temporal and cross-region interactions in a network (17). Compared with hypothesis-driven analysis, the specification of model content in GC lies in the regions of interest (ROIs) involved in a network and the number of lags to use. Once the ROIs are defined, it is the data itself that leads to the statistical inference of temporal and crossregion interactions.

\section{Objectives}

In the current study, we examined the directional interactions among the supplementary motor area (SMA) and M1 area when participants performed action verb reading, motor imagery and hand motions. SMA is a part of the primate cerebral cortex contributing to the control of movement, and M1 is the main area contributing to movement execution. The two regions have been found strongly involved in hand motion execution. In this study, participants passively read verbs about manual action, performed motor imagery of manual action, and executed manual actions. Anatomical ROIs were selected in the bilateral SMA and M1, and then GC analysis was used to compute the causal influences among the four regions. According to strong embodiment (5), the same neural substrates are involved in perceiving/doing, imaging and language comprehension. Thus, the motor system plays similar roles in action language comprehension, motor imagery and action execution. If this is true, then similar connectivity patterns should be observed in all task modes. According to weak embodiment, sensory-motor information partly contributes to semantic processing, and the motor system might play different roles in action verb processing, motor imagery and action execution. If this is true, then the connectivity pattern in action verb processing might show both similarities and differences with those in motor imagery and hand motion execution.

\section{Materials and Methods}

\subsection{Participants}

Participants included 19 Chinese native speakers (7 males, average 24.7 years) who got compensation for the participation. All were right-handed according to a Chinese version of the Edinburgh Handedness Inventory (20). All had normal or corrected to normal vision and none had a history of neurological or psychiatric disease. Each participant provided the written informed consent to the procedures approved by the Imaging Center for Brain Research at Beijing Normal University. The study protocol conforms to the ethical guidelines of the 1975 Declaration of Helsinki as reflected in a priori approval by the institution's human research committee.

\subsection{Materials}

The detailed information about the stimuli in the passive reading task can be found in (21). Here we provided information relevant to the stimuli used in this study. Both the passive reading session and the motor imagery session contained 48 single-character Chinese verbs about manual actions, such as jiao (stir), sao (sweep), wa (dig). All verbs were rated on familiarity, concreteness and imageability with Likert-like 7-point scales $(1=$ very low, 7 = very high) and hand action ratio with a dichotomous scale $(1=$ yes, $0=$ no) by 20 native Chinese speakers (none of them participated in the fMRI experiment). The word frequency of each verb was obtained from the Language Corpus System of Modern Chinese Studies (22). Paired t-tests showed that the manual verbs in the passive reading task and the motor imagery task matched well with each other (ts $<1$, ps $>0.1$ ) (Table 1$)$.

Table 1. Descriptive Statistics of Stimuli Characteristics in the Passive Reading Task and the Motor Imagery Task ${ }^{a}$

\begin{tabular}{lllllc}
\hline Task & Frequency & Familiarity & Concreteness & Imageability & Hand Ratio \\
\hline Passive reading & $31.8 \pm 30.2$ & $5.4 \pm 0.8$ & $5.2 \pm 0.7$ & $5.8 \pm 0.5$ & $0.8 \pm 0.1$ \\
Motor imagery & $32.6 \pm 30.0$ & $5.5 \pm 0.7$ & $5.2 \pm 0.6$ & $5.9 \pm 0.4$ & $0.8 \pm 0.1$ \\
\hline
\end{tabular}

${ }^{\mathrm{a}}$ Hand ratio refers to the hand action ratio. Data are presented as mean \pm SD. 
Yang J et al.

\subsection{Task and Procedure}

The fMRI experiment included three separate task sessions: a passive reading session, a motor imagery session, and a hand motion session. To avoid the influences of motor imagery and hand motion on passive reading, the order of the three tasks was fixed: the passive reading session always preceded the motor imagery session (14), and the hand motion session was always the last one.

The procedures for the passive reading session and the motor imagery session were same. The 48 verbs in each session were divided into 6 blocks, and in each block a verb was displayed on the screen for 2 sec plus a .5 sec blank. Each block was followed by a 16 sec rest. Participants were told that in the passive reading task they should read each verb carefully and that in the motor imagery task they should imagine the situation that they were doing the actions described by each presented verb. In both tasks, participants were asked to keep their head, hand and body still.

The hand motion session contained 6 task blocks, and each block lasted 20 sec followed by a 16 sec rest. Within each block, a signal consisted of three asterisks $\left(^{* * *}\right)$ appeared on the screen 10 times to indicate the frequency of hand motion. Participants were instructed to pantomime grasping actions with their left or right hand according to the frequency of the asterisk signal. An instruction appeared on the screen to tell participants which hand they should use at the beginning of each block. The order of hand motion was randomized.

After scanning, participants performed two post-tests. They were asked to select the words presented in the passive reading session from a word list and select pictures that were similar to their imagery from a picture list. All participants could recognize the presented words and pictures describing depicted actions.

\subsection{Data Acquisition}

Image acquisition was performed at the Imaging Center for Brain Research in Beijing Normal University using a 3.0-T, whole-body MRI system (Siemens, Trio Tim) with a standard radiofrequency head coil. Functional images were acquired with a gradient echo planar imaging (EPI) sequence $(\mathrm{FA}=900, \mathrm{TR}=2000 \mathrm{~ms}, \mathrm{TE}=30 \mathrm{~ms}, 32$ axial slices, thickness $=4 \mathrm{~mm}$, inter-slice gap $=8 \mathrm{~mm}$, voxel dimension $=3.125 \times 3.125 \times 4.8 \mathrm{~mm}^{3}, 32$ axial slices). After that, high-resolution anatomical MRI for each participant was acquired using a MPRAGE sequence $(F A=70 ; \mathrm{TR}=2530$ $\mathrm{ms}, \mathrm{TE}=3.39 \mathrm{~ms}, 128$ sagittal slices, voxel dimension $=1.33$ $\times 1.33 \times 1.33 \mathrm{~mm}^{3}$ ).

\subsection{Data Analysis}

The fMRI data were analyzed with AFNI software package (23). Head motion correction for EPI images was performed with a six-parameter rigid-body transformation after slice timing. Each participant's anatomical image was coregistered to standard Talaiarach and Tournoux space (24), and the EPI images were aligned to the anatomical image. A 6-mm Gaussian kernel was used to spatially smooth the functional data and all images were resampled to $2 \times 2 \times 2 \mathrm{~mm}^{3}$ resolution.

A Granger Causality analysis was conducted to investigate the causal relations among the bilateral M1 and SMA. Anatomical ROIs in the bilateral M1 were selected based on the TT-Damon Template, and anatomical ROIs in the bilateral SMA were selected based on the TT-N27 template (Figure 1). After that, the average time series in each ROI was computed for each participant. 1dGC.R program (http://afni.nimh.nih.gov/sscc/gangc/1dGC) in the AFNI package was employed to conduct the GC analysis. In individual analysis, the averaged time series in each ROI was entered into a VAR model as the input. The model was chosen according to the Akaike Information Criterion. Six head-motion parameters were entered into the VAR model as covariates to minimize confounding effects. For each participant, both the path coefficients and the corresponding t-values for the causal effects among the four ROIs were computed. Group analysis was conducted based on the path coefficients and the t-values from individual analysis. Group t-tests were used to test the significance of the causal relations among the ROIs. The final results were reported at the threshold of $\mathrm{P}<0.05$ with Bonferroni correction for multiple comparisons.

\section{Results}

\subsection{Behavioral Results}

Accuracies of word recognition and picture recognition were expressed as scores. In the passive reading task, the average d-prime was $2.57(\mathrm{SD}=0.90)$. In the motor imagery task, the overall average d-prime was $2.37(\mathrm{SD}=0.80)$.

Figure 1. Mask of ROIs in the Bilateral Supplementary Motor Areas and Bilateral Primary Motor Cortex

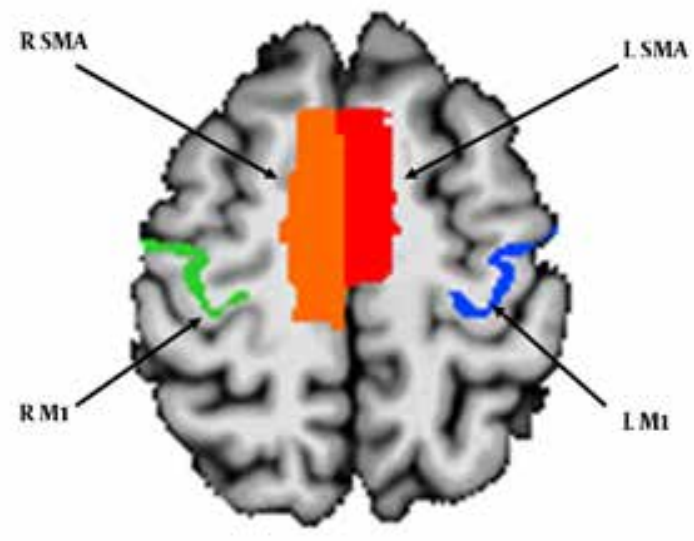

L, left; R, right; M1, primary motor cortex; SMA, supplementary motor area 
No significant task effect was revealed by paired-sampled t-test $[\mathrm{t}(18)=0.757, \mathrm{P}=0.458]$.

\subsection{GC Results}

\subsubsection{GC Results in the Passive Reading Task}

In the passive reading task, the left M1 showed influences on the bilateral SMA, and bidirectional influences was found between the left M1 and the right M1 (Figure $2 \mathrm{~A}$ ).

\subsubsection{GC Results in the Motor Imagery Task}

In the motor imagery task, the causal interactions among the four ROIs were more complicated as compared with that in the passive reading task. The left M1 showed an influence on the left SMA, and bidirectional influences were indicated between the bilateral M1. In addition, the left SMA and the right M1 indicated influences on the right SMA, and the right SMA showed negative influences on the bilateral M1 (Figure 2 B).

\subsubsection{GC Results in the Hand Motion Task}

In the hand motion task, complicated causal interactions were found among the bilateral M1 and SMA (Figure $2 \mathrm{C}$ ). The bilateral M1 and left SMA showed positive influences on the right SMA, while the right SMA indicated negative influences on the other three regions. Additionally, the bilateral M1 showed influence on the left SMA.

Figure 2. Granger Causality Results for the Three Tasks

A

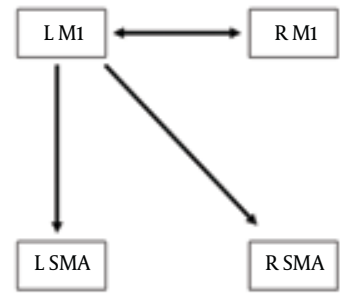

B

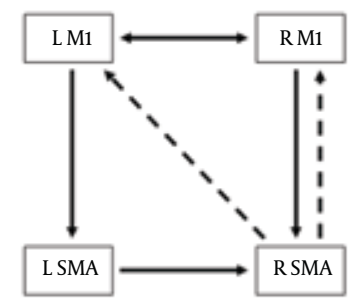

C

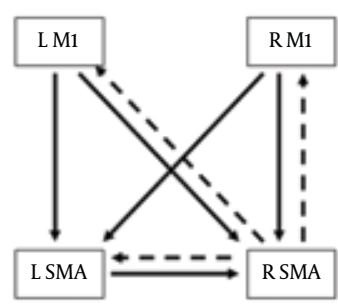

(A) Granger causality results for the passive reading task. (B) Granger causality results for the motor imagery task. (C) Granger causality results for the hand motion execution task. Single directional arrows indicate single directional causal influences, and bidirectional arrows indicate bidirectional causal influences. Solid lines indicate positive influences. Dashed lines indicate negative influences. $\mathrm{L}=$ left, $\mathrm{R}=$ right, $\mathrm{M} 1$ = primary motor cortex, SMA = supplementary motor area.

\section{Discussion}

The embodied feature of semantic representation is still in controversy. Previous studies have shown different activations in the motor system during action verb processing and motor imagery; however, whether the motor system interaction is modulated by task demand is still unknown. The current study used effective connectivity analysis to investigate the causal interactions among the bilateral SMA and M1 during verb passive reading, motor imagery, and hand motion execution. The results indicated that the complexity of the connectivity pattern changed across tasks: The hand motion task elicited the most complicated network, the motor imagery task elicited a less complicated network, and the passive reading task elicited the fewest connections. A similar connectivity was found between the left M1 and left SMA in all tasks. Additionally, passive reading and motor imagery indicated similar connectivity patterns between the bilateral M1, and similar connectivity patterns between the left M1 and right SMA was revealed in passive reading and the hand motion task. Finally, similar negative influences from the right SMA on other regions were revealed in motor imagery and the hand motion task.

The most interesting result is the common connectivity between left M1 and left SMA across all three tasks. An influence from the left M1 to left SMA was observed. Previous studies found that SMA is important in storing the information necessary for the orderly performance of multiple movements and planning movements ahead (25), and that SMA is strongly activated when participants imagined that they were performing a complex sequence of finger movements (26). The findings suggest that SMA might be involved in 'high-order' aspects of motor behavior (27-30), such as the internal generation of complex movements $(26,27,31)$. Recent studies also show that SMA activation can be tightly coupled to M1 during externally cued movements (32). In the current study, a directional influence was found from M1 to SMA during three tasks, and this might suggest that the possible sequence of hand movements cued by manual action verbs during passive reading and motor imagery and the sequence information of hand movements in the motion execution task are processed and stored by SMA to generating the plan of incoming movements (25).

Another interesting result about the connectivity between SMA and M1 is that in the motor imagery and the hand motion tasks, causal influences were found from M1 to SMA bilaterally, but in the passive reading task such influence was only found in the left hemisphere. Given the fact that all the participants in the current study are right-handed, the results suggest that motor component in the semantic representation of manual verbs can be body-specific and shaped by actions one has performed (1). This finding is consistent with (14) which found that handedness can influence motor activity during hand verb comprehension. While right-handed participants 
YangJ et al.

activated the left premotor areas during lexical decision of hand action verbs, left-handed participants activated the right premotor areas.

Besides the similar connectivity found between the left M1 and left SMA in all three tasks, the passive reading task also indicated similar bilateral connectivity between the left M1 and right M1. But such connectivity patterns was not revealed in the hand motion task. This difference might relate to the stimuli used in the experiment. In both the passive reading and the motor imagery tasks, the manual verbs described complex hand actions. Some of the actions require tool-use, such as qie (cut), kan (chop) and jiao (stir). These complex manual actions can involve both hands, and this might induce the connectivity between the bilateral M1. In the hand motion task, however, the grasping actions performed by participants merely require one hand. Thus, the connectivity between M1 and SMA was observed in each hemisphere, but no connectivity was found between the bilateral M1.

One important difference between the passive reading results and the motor imagery / hand motion results is that the negative influences from the right SMA were only found in the latter two tasks. Several studies have investigated the connectivity between SMA and other motor areas during motor imagery $(3,33)$, and they have demonstrated that SMA has a suppressive influence on M1 during motor imagery $(3,33)$. In Solodkin et al., participants performed kinetic imagery (i.e. mental simulation of movement associated with a kinesthetic feeling), visual imagery (i.e. visual representation of their moving limbs) of manual actions and executed manual actions. The structural equation modelling results showed that the connection from SMA to M1 became suppressive during kinesthetic motor imagery (33). This result suggests a physiological mechanism through which the motor system prevents overt movements. Kasess et al. utilized dynamic causal modeling to determine the effective connectivity between SMA and M1 and they found a strong suppressive influence from SMA to M1 in the motor imagery condition (3). This finding indicated that SMA is important for the preparation and suppression of movements. In the current study, both motor imagery and motion execution tasks showed that the right SMA had suppressive influences on the bilateral M1. This is consistent with previous findings that SMA in each hemisphere is reciprocally connected and projects to both contralateral and ipsilateral M1 $(34,35)$, and that SMA can operate bilaterally (36). However, the suppressive influences from SMA were only found in the right hemisphere, suggesting that the bilateral SMA might play different roles in motor execution and motor imagery. Another result that might support this view is that in the motor imagery and motor execution tasks, the right SMA received positive influences from other seed regions including the left SMA (Figure $2 \mathrm{~B}$ and 2C). Previous studies have found that when participants had a hand preference, the involvements of the bilateral SMA during hand or finger movements can be different (37). Given the fact that all participants in this study were right-handed, whether handedness influences the role of the bilateral SMA in motor execution and motor imagery needs further investigation.

One methodological issue in the current study is the GC modeling used for computing effective connectivity. Smith et al. claimed that hemodynamic variability between different brain regions may swamp any causal lag in the underlying neural time series, and thus cause bias in lag-based causality analysis (e.g., GC analysis) (38). According to Roebroeck et al., one possible approach to exclude the confound effect caused by systematic difference in the hemodynamic lag at two regions is to show the influence varies in different experimental conditions or cognitive contexts (39). In the present study, we found that the causal interactions varied in different cognitive contexts (i.e. verb passive reading, motor imagery and motion execution) and thus the current result cannot be interpreted merely by the hemodynamic variability between SMA and M1. However, there is still an issue that in GC analysis the instantaneous correlation among regions in a network is regarded to be irrelevant (17), and thus the information of the contemporaneous interactions among the bilateral SMA and bilateral M1 were lost. Future work should combine GC analysis with connectivity analysis focusing on the instantaneous correlations between regions to explore the causal influences within the networks for different task demands.

To summarize, the current finding indicate that although the motor network involved in action verb processing shares similar interactions with those in motor imagery and hand motion execution, the network is less complicated. This result pattern suggests that semantic representation might share some common features with motor imagery and motor execution, but the neural mechanisms under these processes are different. The current result supports weak embodiment which claims that the motor system can be partly involved in and contribute to the semantic representation of action language processing, but semantic representation is different from the raw sensory-motor experiences supported by the primary cortical areas $(6,7)$.

\section{Acknowledgements}

None declared.

\section{Authors' Contributions}

Study concept and design: Jie Yang and Hua Shu; Analysis and interpretation of data: Jie Yang; Drafting of the manuscript: Jie Yang; Critical revision of the manuscript for important intellectual content: Jie Yang; Statistical analysis: Jie Yang.

\section{Financial Disclosure}

The authors declared that they had no conflicts of inter- 
est that could inappropriately influence, or be perceived to influence, their work.

\section{Funding/Support}

This research was supported by the Program for Changjiang Scholars and Innovative Research Team in University (IRT0710).

\section{References}

1. Meteyard L, Cuadrado SR, Bahrami B, Vigliocco G. Coming of age: a review of embodiment and the neuroscience of semantics. Cortex. 2012;48(7):788-804.

2. Glenberg AM, Robertson DA. Symbol Grounding and Meaning: A Comparison of High-Dimensional and Embodied Theories of Meaning. J Mem Lang. 2000;43(3):379-401.

3. Kasess $\mathrm{CH}$, Windischberger $\mathrm{C}$, Cunnington $\mathrm{R}$, Lanzenberger $\mathrm{R}$, Pezawas L, Moser E. The suppressive influence of SMA on M1 in motor imagery revealed by fMRI and dynamic causal modeling. Neuroimage. 2008;40(2):828-37.

4. Zwaan RA. The immersed experiencer: Toward an embodied theory of language comprehension. Psychol Learn Motiv. 2004;44:35-62.

5. Gallese V, Lakoff G. The Brain's concepts: the role of the Sensorymotor system in conceptual knowledge. Cogn Neuropsychol. 2005;22(3):455-79.

6. Simmons WK, Barsalou LW. The similarity-in-topography principle: reconciling theories of conceptual deficits. Cogn Neuropsychol.2003;20(3):451-86.

7. Vigliocco G, Vinson DP, Lewis W, Garrett MF. Representing the meanings of object and action words: the featural and unitary semantic space hypothesis. Cogn Psychol. 2004;48(4):422-88.

8. de Zubicaray G, Postle N, McMahon K, Meredith M, Ashton R. Mirror neurons, the representation of word meaning, and the foot of the third left frontal convolution. Brain Lang. 2010;112(1):77-84.

9. Fernandino L, Iacoboni M. Are cortical motor maps based on body parts or coordinated actions? Implications for embodied semantics. Brain Lang. 2010;112(1):44-53.

10. Hauk O, Johnsrude I, Pulvermuller F. Somatotopic representation of action words in human motor and premotor cortex. Neuron. 2004;41(2):301-7.

11. Kemmerer D, Castillo JG, Talavage T, Patterson S, Wiley C. Neuroanatomical distribution of five semantic components of verbs: evidence from fMRI. Brain Lang. 2008;107(1):16-43.

12. Postle N, McMahon KL, Ashton R, Meredith M, de Zubicaray GI. Action word meaning representations in cytoarchitectonically defined primary and premotor cortices. Neuroimage. 2008;43(3):634-44.

13. Pulvermuller F, Hauk O, Nikulin VV, Ilmoniemi RJ. Functional links between motor and language systems. Eur J Neurosci. 2005;21(3):793-7.

14. Willems RM, Hagoort P, Casasanto D. Body-specific representations of action verbs: neural evidence from right- and left-handers. Psychol Sci. 2010;21(1):67-74.

15. Friston KJ, Ungerleider LG, Jezzard P, Turner R. Characterizing modulatory interactions between areas V1 and V2 in human cortex: A new treatment of functional MRI data. Hum Brain Mapp. 1994;2(4):211-24.

16. Bollen KA. Structural Equations with Latent Variables.: Wiley; 1999.

17. Chen G, Glen DR, Saad ZS, Paul Hamilton J, Thomason ME, Gotlib $\mathrm{IH}$, et al. Vector autoregression, structural equation modeling, and their synthesis in neuroimaging data analysis. Comput Biol Med.2011;41(12):1142-55.

18. Cooper RA, Boninger ML, Spaeth DM, Ding D, Guo S, Koontz AM, et al. Engineering better wheelchairs to enhance community participation. IEEE Trans Neural Syst Rehabil Eng. 2006;14(4):438-55.

19. Granger CWJ. Investigating causal relations by econometric models and cross-spectral methods. Econometrica. 1969:424-38.

20. Oldfield RC. The assessment and analysis of handedness: the Edinburgh inventory. Neuropsychologia.1971;9(1):97-113.

21. Yang J, Shu H, Bi Y, Liu Y, Wang X. Dissociation and association of the embodied representation of tool-use verbs and hand verbs: An fMRI study. Brain Lang. 2011;119(3):167-74.

22. Sun HL, Huang JP, Sun DJ, Li DJ, Xing H. B. . Introduction to Language Corpus System of Modern Chinese Study. In: Hu M. Y editor. Paper collection for the Fifth World Chinese Teaching Symposium.. Beijing: Peking University Publisher; 1997. pp. 459-66.

23. Cox RW. AFNI: software for analysis and visualization of functional magnetic resonance neuroimages. Comput Biomed Res. 1996;29(3):162-73.

24. Talairach J, Tournoux P. Co-planar stereotaxic atlas of the human brain.New York: Thieme Medical; 1988.

25. Tanji J, Shima K. Role for supplementary motor area cells in planning several movements ahead. Nature.1994;371(6496):413-6.

26. Roland PE, Larsen B, Lassen NA, Skinhoj E. Supplementary motor area and other cortical areas in organization of voluntary movements in man. J Neurophysiol.1980;43(1):118-36.

27. Goldberg G. Supplementary motor area structure and function: review and hypotheses. Behav Brain Sci.1985;8(4):567-88.

28. Picard N, Strick PL. Motor areas of the medial wall: a review of their location and functional activation. Cereb Cortex 1996;6(3):342-53.

29. Tanji J. The supplementary motor area in the cerebral cortex Neurosci Res. 1994;19(3):251-68.

30. Tanji J. Sequential organization of multiple movements: involvement of cortical motor areas. Annu Rev Neurosci. 2001;24:631-51.

31. Orgogozo JM, Larsen B. Activation of the supplementary motor area during voluntary movement in man suggests it works as a supramotor area. Science.1979;206(4420):847-50.

32. Picard N, Strick PL. Activation of the supplementary motor area (SMA) during performance of visually guided movements. Cereb Cortex. 2003;13(9):977-86.

33. Solodkin A, Hlustik P, Chen EE, Small SL. Fine modulation in network activation during motor execution and motor imagery. Cereb Cortex. 2004;14(11):1246-55.

34. Muakkassa KF, Strick PL. Frontal lobe inputs to primate motor cortex: evidence for four somatotopically organized 'premotor' areas. Brain Res. 1979;177(1):176-82.

35. Pandya DN, Vignolo LA. Intra- and interhemispheric projections of the precentral, premotor and arcuate areas in the rhesus monkey. Brain Res. 1971;26(2):217-33.

36. Deecke L. Bereitschaftspotential as an indicator of movement preparation in supplementary motor area and motor cortex. Ciba Found Symp. 1987;132:231-50.

37. Solodkin A, Hlustik P, Noll DC, Small SL. Lateralization of motor circuits and handedness during finger movements. Eur J Neurol. 2001;8(5):425-34

38. Smith SM, Miller KL, Salimi-Khorshidi G, Webster M, Beckmann CF, Nichols TE, et al. Network modelling methods for FMRI. Neuroimage. 2011;54(2):875-91.

39. Roebroeck A, Formisano E, Goebel R. Mapping directed influence over the brain using Granger causality and fMRI. Neuroimage. 2005;25(1):230-42. 